PACS: $42.79 . \mathrm{Kr}$

\title{
Development and investigation of LC light shutters for a welding automatic mask
}

\author{
Yu. Kolomzarov, P. Oleksenko, V. Sorokin, P. Tytarenko, R. Zelinskyy \\ V. Lashkaryov Institute of Semiconductor Physics, NAS of Ukraine, 41, prospect Nauky, 03028 Kyiv, Ukraine \\ Phone: 525-3126. Fax: 525-5785. \\ E-mail: zelinsky@isp.kiev.ua
}

\begin{abstract}
Elements of technology to produce liquid crystal (LC) light shutters for a welding automatic mask with the built-in transparent heater for operating LC layer were developed. Experimental samples of light shutters were created using the developed technology, and their parameters and characteristics were investigated. Measuring the "switch-on" times of the created light shutters has shown that the transition from the transparent state to the opaque one within the temperature range from -20 up to $+50{ }^{\circ} \mathrm{C}$ lasts less than $1.5 \mathrm{~ms}$. This value meets the requirements of international standards.
\end{abstract}

Keywords: light shutter, welding mask, liquid crystal.

Manuscript received 06.08.04; accepted for publication 16.12.04

\section{Introduction}

Wide application of artificial high-powered light sources both in industry and in private life, and extending progression of electrowelding technology requires to solve actual problems of sight protection from bright light flashes. The essence of all the methods for protection of human eyes from bright light flashes is based on light radiation attenuation up to retain the levels fixed by medico-biologic requirements. Technical achievements of automatic vision protection methods are based on the different laws of mechanics, physics, chemistry, etc. For instance, some years ago the photochromic and thermophotochromic light shutters were developed [1]. In foreign and domestic literature $[2,3]$, the experimental samples of electromechanical light shutters are described. However, as seen from our literature analysis, the most successful and fast acting are electrooptical light shutters $[4,5]$. Among them, the LC light shutters hold a special place. Due to the fact that LC light shutters demonstrate the high response speed $\left(10^{-3} \mathrm{~s}\right)$ and the possibility to attenuate light emission up to $10^{3}$ times and more, they are widely used for creation of automatic light shutters for welding masks (WM) abroad. Several years ago, some companies of USA, Germany, Sweden, Switzerland, Hungary, Poland, Japan, and China started production of LC automatic light shutters for WM. Representatives of these companies consider that such equipment is caused by revolutionary changes in technique and technology of electrowelding, and its high cost is reasonable $[6,7]$.

Up to this time in Ukraine and another Former Soviet Union countries, the production of above- mentioned vision protection facilities was unavailable. In foreign scientific-technical literature, there is the detailed information on the possibilities to use LC light shutters for WM creation, but some questions on construction and production technology are not published and are classified as "know-how". Taking into account the importance and urgency of this problem, we carried out investigations in development and production of automatic LC light shutters for WM [8].

The main idea of such facility construction is based on implementation of an automatic control by the transmission coefficient depending on the emission power of electrical arc. At the same time, the transition time from the transparent to opaque state must lie within the range $0.5 \ldots .5 \mathrm{~ms}$ according to the requirements of international standards. Construction of such facilities with the electrooptical effect occurring in LC gives a possibility to develop the relatively low-cost and simple to operate vision protection devices.

In this article, we demonstrate the results of the development of LC light shutters with built-in transparent heater under arduous temperature operating conditions. We also compared its characteristics with those of foreign prototypes.

\section{Experimental setup}

As the result of deep analysis of literature sources we selected twist effect in nematic LC as electrooptical effect in LC. It is known that switching on time $\tau_{\text {on }}$ and switching off time $\tau_{\text {off }}$ of twist effect depend on the applied voltage $U$, the thickness of the LC layer $d$, the rotational viscosity of the LC composition $\eta$, Frank 


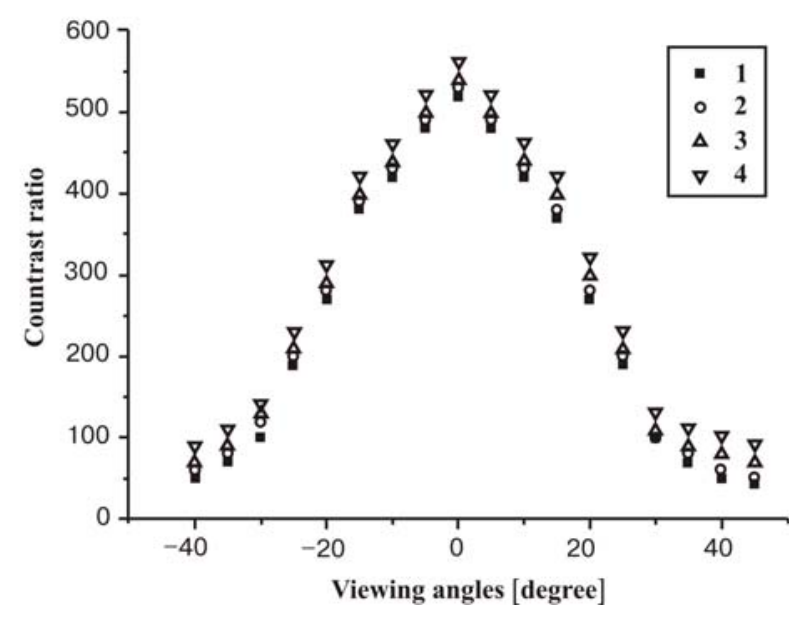

Fig. 1. Dependence of the contrast ratio vs angles in the horizontal plane of view at the ambient temperature $20^{\circ} \mathrm{C}$ for 4 LC light shutters.

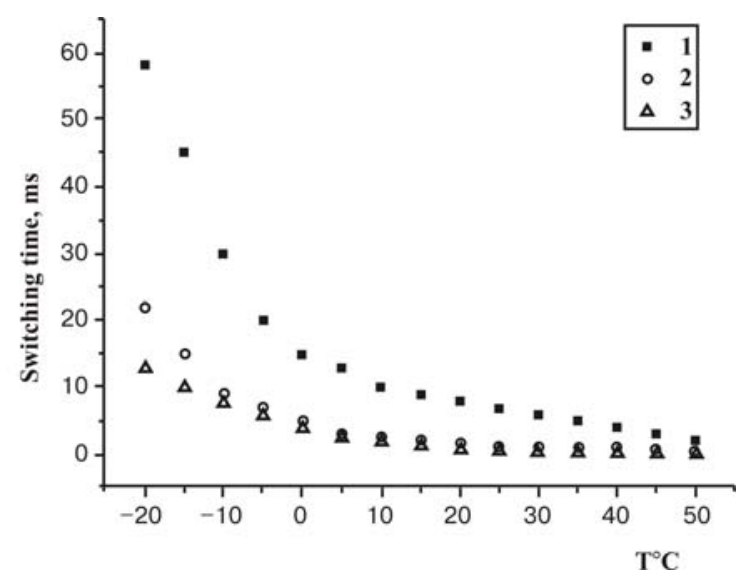

Fig. 2. Dependence of the switch-on time vs ambient temperatures for LC light shutters without heating.

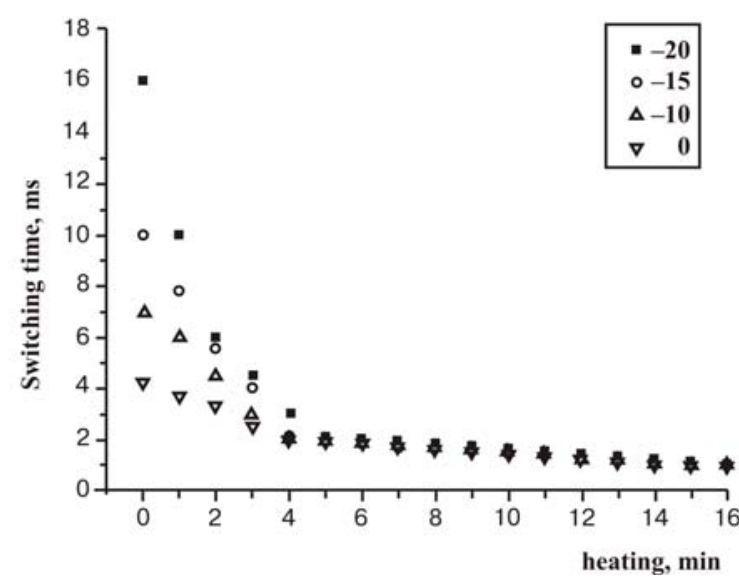

Fig. 3. Dependence of the switch-on time vs heating duration for LC light shutters at different ambient temperatures. elastic constants of LC material $K_{11}, K_{22}$, and $K_{33}$, and the dielectric anisotropy of the LC material $\Delta \varepsilon$ which are given by the following equations $[9,10]$ :

$\tau_{\text {on }}=\frac{\eta d^{2}}{\left(\frac{\Delta \varepsilon U^{2}}{4 \pi}-K \pi^{2}\right)}$,

$\tau_{\text {off }}=\frac{\eta d^{2}}{K \pi^{2}}$,

$K=K_{11}+\frac{K_{33}-K_{22}}{4}$

Calculations of switching times showed that, for a wide class of LC materials such as LC 1289, LC 1285, LC 1630A, and LC 1391, values of $\tau_{\text {on }}$ and $\tau_{\text {off }}$ were larger than $10 \mathrm{~ms}$ when the temperature was within the range from 20 to $40{ }^{\circ} \mathrm{C}$, the applied voltage was $15 \mathrm{~V}$ and the LC thickness was from 10 to $20 \mu \mathrm{m}$. When the temperature was higher than $40^{\circ} \mathrm{C}$, it was possible to decrease $\tau_{\text {on }}$ down to $5 \mathrm{~ms}$. It can be explained by the temperature behavior of the rotational viscosity $\eta$. It is known that the rotational viscosity coefficient $\eta$ of LC material (as for common liquid) satisfies the Andrade formula given below:

$\eta=A e^{\frac{B}{T}}$

where $A$ and $B$ are constants, and $T$ is the absolute temperature. It can be seen from the expression that the rotational viscosity $\eta$ decreases exponentially with increasing the temperature. Moreover, it can be seen that temperature adjusting is effective in improving the response speed of LC display apparatus. Other physical properties of the LC material, e.g., $\Delta \varepsilon$ and $\Delta n$, change in a way that the anisotropy thereof decreases as the temperature increases, as does the order parameter $S$. Therefore, a comprehensive design that takes into consideration the temperature dependence on various physical properties is necessary. From the viewpoint of switching times, it is believed to be the most effective to design the LC panel focusing on the rotational viscosity while adjusting the optical and electrical anisotropy dependent on the temperature.

For given electrophysical properties of LC materials, the operation voltage and temperature ranges only decreasing the thickness of LC layer can reduce the switching time. However, for creation of LC light shutter with a high contrast ratio when LC layer thickness is less than $10 \mu \mathrm{m}$, it is necessary to take into consideration the light transmission dependence on LC layer thickness which is given by the Gouchi - Tarri formula: 


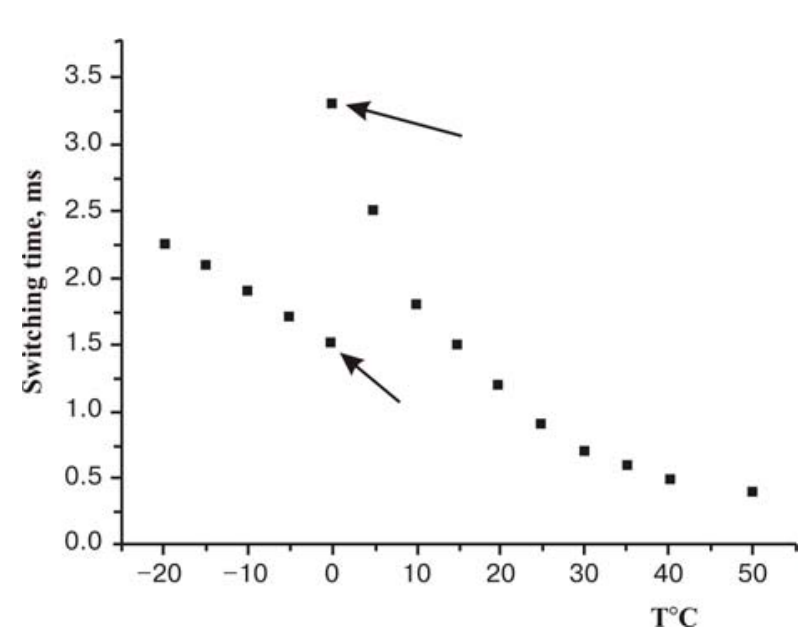

Fig. 4. Dependence of the switch-on time vs ambient temperatures for LC light shutters. Heating the system was switched on when the ambient temperature become below zero. The upper arrow corresponds to the start of heating, and the lower arrow - to the ready state of LC light shutter.

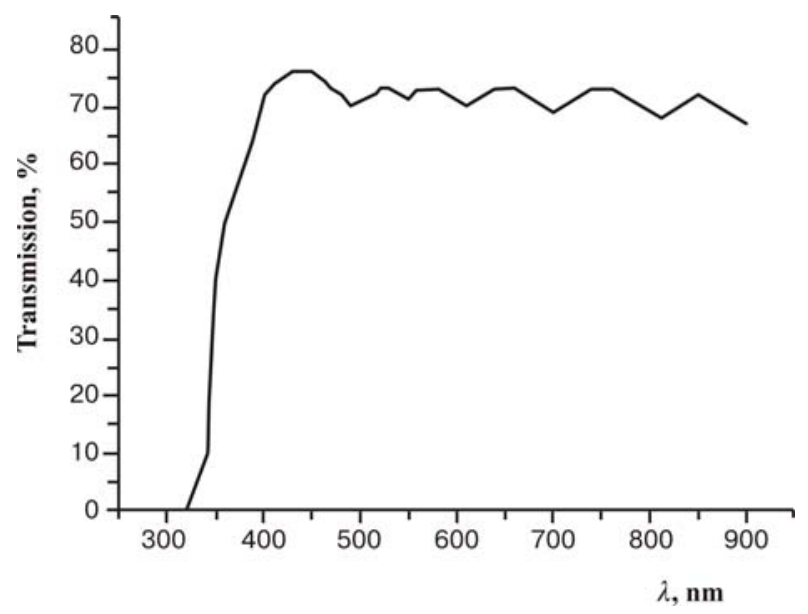

Fig. 5. Transmission spectrum of a LC light shutter in the transparent state.

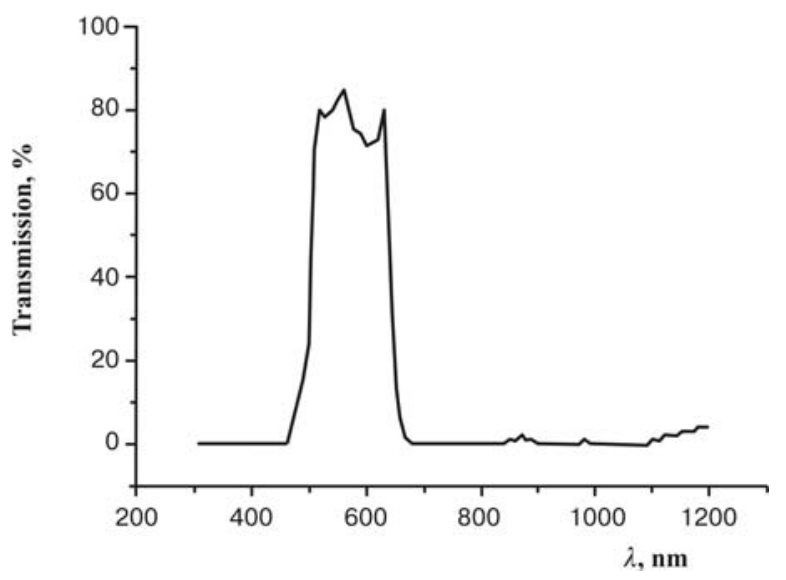

Fig. 6. Transmission spectrum of the selective interference light filter.

$$
\begin{aligned}
& I=I_{0}\left(1-\frac{\sin (2 \varphi)\left(1+U^{2}\right)^{\frac{1}{2}}}{1+U^{2}}\right), \\
& U=\frac{\pi d\left(\frac{n_{e}}{1+\omega \sin \left(2 \Theta_{s}\right)^{\frac{1}{2}}}\right)-n_{o}}{\varphi \lambda}
\end{aligned}
$$

where $\varphi$ is the twist angle, $\omega=\frac{n_{e}}{n_{o}}, \lambda$ is the wavelength of the transmitted light, $\Theta_{s}$ is the tilt angle of the LC molecules on the substrate surface.

For the selection of optimal LC material, the comparison analysis of electrophysical parameters LC materials was carried out. Taking into account the specific operation conditions of vision protection facilities and requirement of minimum of $\tau_{\text {on }}$ value, the most optimal materials LC 1630A and LC 1289 were used. But LC 1289 is more preferable because of the following reasons:

- material LC $1630 \mathrm{~A}$ is experimental (this material is not available in commercial production) and characterized as unstable by its physical parameters under operating conditions till to full inoperativity;

- material LC 1289 has a specification, it is an apprais material. By such parameters as the rotational viscosity coefficient and the operation temperature range, LC 1289 is completely adequate to the requirements upon LC light shutters for WM. Results of the theoretical calculations and the experimental investigations showed that, for LC 1289 material, the optimal thickness of LC layer must be $5.0 \pm 0.1 \mu \mathrm{m}$.

\section{Results and discussion}

Developed automatic LC light shutter is a monolithic construction and consists of one or two LC light shutters (depending on the class of the welding light filter). LC light shutter has a transparent heater of LC layer. The electronic control circuit switches on the heater automatically, when the ambient temperature sinks below zero.

The design feature of LC light shutter technology consists in using inorganic $\mathrm{SiO}_{\mathrm{x}}$ films as orienting layers deposited by the method of reactive cathode sputtering $[11,12]$. It makes possible to increase the modulation depth of LC light shutter, to enlarge the circular contrast indicatrix, and to reduce the shutter power consumption through increasing of operating frequency of fractions of $\mathrm{Hz}$ (under operating conditions, when the ambient temperature is that it does not require heating the LC light shutter).

Investigation of dependences of the contrast ratio vs applied voltage showed that this ratio has a maximum at 
the applied voltage equal to $10 \mathrm{~V}$. When the voltages are higher than $10 \mathrm{~V}$, the contrast ratio becomes stable and only $\tau_{\text {on }}$ value decreases. When the applied voltages are higher than $16 \mathrm{~V}$, the contrast ratio, at normal viewing, has values from 550:1 up to 580:1 for one LC light shutter. At the same time, two optically connected series LC light shutters can demonstrate the contrast ratio approximately $300,000: 1$.

Investigation of dependences of the contrast ratio vs viewing angle showed that technological methods for creation of orienting structures allow to control the contrast indicatrix. Developed LC light shutters have a maximal contrast ratio in the horizontal plane of view. The maximum contrast ratio in the horizontal plane of view is guaranteed by orientation of LC molecules along the short edge of one glass substrate and long edge of another glass substrate with shifting by $3^{\circ}$. Characteristics of the contrast ratio vs viewing angles for four samples of LC light shutter are shown in Fig. 1. All the characteristics of the contrast ratio vs viewing angles coincide, which confirms the high repeatability of technological processes. For the viewing angles $\pm 10^{\circ}$, the contrast ratio is larger than 150:1 and, for viewing angles $\pm 35^{\circ}$, it exceeds 70:1. These values correspond to the international standard requirements.

In Fig. 2, the temperature dependences of the switching-on time $\tau_{\text {on }}$ for developed LC light shutter and two compatible LC light shutters produced abroad are shown. Temperature dependences of the switching-on time for all the samples are approximately the same. This fact indicates the necessity of heating the LC layer to obtain the switch-on time values less than $5 \mathrm{~ms}$ at the ambient temperatures below zero.

In Fig. 3, the dependences of the switch-on time vs duration of heating for different ambient temperatures when the optimal electrical power is applied to the transparent heater are shown. As we can see from Fig. 3, the LC light shutter demonstrates required switching times 3 min later switch on the heater over the ambient temperature range from $-20^{\circ} \mathrm{C}$ up to zero. Optimization of the transparent heater power allows us to exclude using the complicated electronic systems for thermal stabilization and simplifies the heater control electronic circuit.

Fig. 4 demonstrates results of measuring the switch-on time of the LC light shutter with heating system for ambient temperatures over the range of -20 to $+50{ }^{\circ} \mathrm{C}$.

Spectral characteristics of the welding mask separate parts such as LC light shutter (see Fig. 5) and a specially developed selective interference light filter (see Fig. 6) demonstrate the following optical parameters of AWM in the transparent and opaque states.
Main parameters of AWM measured in the transmission mode in the opaque state:

Class number of AWM according

to OST 21-6-87

Identification of AWM according to OST 21-6-87

Transmission, $\%$

- UV region at $\lambda=320 \mathrm{~nm}$

- visible region at $\lambda=550 \mathrm{~nm}$

- IR region at $\lambda=1000 \mathrm{~nm}$

\section{Main parameters AWM measured in the} transmission mode in the transparent state: Class number of AWM according to DIN 4647 (EN 379)

less than 4

Transmission, $\%$

- UV region at $\lambda=320 \mathrm{~nm}$

0.0001

- visible region at $\lambda=550 \mathrm{~nm}$

- IR region at $\lambda=1000 \mathrm{~nm}$

\section{Conclusions}

The experimental results obtained have shown that the developed AWM consists of one LC light shutter corresponding to 9.5 class number in the opaque state and to 4 class number in the transparent one. Using two LC light shutters, we can increase the AWM class number in the opaque state up to 12. Spectral and switch-on time characteristics meet the requirements of international standards.

\section{References}

1. V.A. Barychevski, G.I. Lashkov, V.A. Cehomski, Photochromism and Application, Chemistry, Moscow (1977).

2. Yu.I. Ivanov, Protection organs of vision from bright light // Foreign Military Review 7, p. 55-61 (1976).

3. V.I. Utkin, Protection glasses // Ibid. 10, p. 67-68 (1978).

4. A.A. Vasil'ev, D.I. Kasasent, I.N. Kompanets, L.V. Parfenov, Spatial time light modulators, Radio and communication, Moscow (1987).

5. M.F. Grebenkin, A.V. Ivashenko, Liquid crystal materials, Chemistry, Moscow (1989).

6. R.M. Pivovar, A.S. Snurnikov, Welding filters with variable darkening // Automatic Welding 1, p. 40-44 (1997).

7. R.M. Pivovar, A.S. Snurnikov, Protection organs of vision from bright light // Science and Defensive 1, p. 50-55 (1998) 
8. V. Sorokin, R. Zelinskyy, Yu. Kolomzarov, A. Chehovoy, V. Belyy, LC light shutters for protection organs of vision from bright light // Proc. $5^{\text {th }}$ Intern. Symp. on Information Displays, Minsk, p.182-185 (1996).

9. S. Palmer, The Optical Properties of Automatically Darkening Welding Filters Based on Liquid Crystal Technology, Acta Universitatis upsalinsis, Upsala (1998).

10. L.M. Blinov, V.G. Chigrinov, Electrooptic effects in liquid materials, Springer-Verlag, New York (1994).
11. Yu. Kolomzarov, P. Oleksenko, V. Sorokin, P. Tytarenko, R. Zelinskyy, Oblique reactive cathode sputtering as a method for creation of orienting liquid crystal microrelief // Proc. $12^{\text {th }}$ Intern. Symposium "Advance Display Technologie", Korolyov, Moskow Region, Russia, August 25-28, p. 150-153 (2003).

12. Yu. Kolomzarov, P. Oleksenko, V. Sorokin, P. Tytarenko, R. Zelinskyy, Vacuum method for creation of liquid crystal orienting microrelief // Semiconductor Physics, Quantum Electronics and Optoelectronics 6 (4), p. 528-532 (2003). 